\author{
Military Technical College \\ Kobry El-Kobbah, \\ Cairo, Egypt.
}

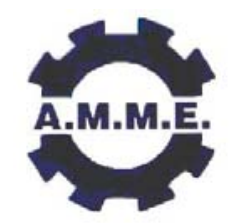

$13^{\text {th }}$ International Conference on Applied Mechanics and Mechanical Engineering.

\title{
ON THE NON-ISENTROPIC SOUND WAVES PROPAGATION OF A STATIONAY OR FLOWING FLUID IN A CYLINDERICAL TUBE FILLED WITH POROUS MEDIA
}

\author{
DUWAIRI* H.M.
}

\begin{abstract}
It is shown that the five parameters governing the propagation of sound waves in a fluid contained in rigid cylindrical tubes filled with a saturated porous media are the shear wave number $s=R \sqrt{\bar{\rho} \omega / \mu}$, ratio of specific heats $\gamma$, the Prandtl number $\sigma$, porosity $\varepsilon$ and the Darcy number $D a=R^{2} / K$. A variational solution of the problem with nonisentropic wave's propagation in a cylindrical tube in the presence and absence of a convective steady flow is presented. The manner in which the flow influences the attenuation and the phase velocity of the forward and backward propagating acoustic waves is deduced. It is found that the inclusion of the solid matrix or the increasing of Darcy number increases the attenuation and decreases the phase velocities of the forward, backward and hydrodynamic sound wave's; this is due to favorable retarding effect of the solid matrix. The increasing of porosity is found to decrease attenuation and increases phase velocities of the forward, backward and hydrodynamic sound waves; this is due to absent role of solid matrix in damping sound waves. The effect of increasing Mach numbers is found to increase attenuation and decrease phase velocities for the backward sound waves; this is due favorable retarding steady flow velocities and decrease attenuation and increase phase velocities of the forward and hydrodynamic sound waves; this is due to favorable steady flow velocities in driving these two sound waves.
\end{abstract}

\section{KEY WORDS}

non-isentropic, sound waves, porous medium, fluid flow

* Mechanical Engineering Department, Faculty of Engineering \& Technology, The University of Jordan, 11942 Amman, Jordan. Email: duwairi@ju.edu.jo

* The work is done during a sabbatical leave year granted to Dr. Hamzeh Duwairi from the University of Jordan for the academic year 2007/2008 at the German Jordanian University. 


\section{NOMENCLATURE}

$\bar{a} \quad$ Mean speed of sound of steady flow

C Constant defined in equation (19)

Da Darcy number, $R^{2} / K$

$i \quad$ Imaginary number, $\sqrt{-1}$

$K \quad$ Permeability

$k \quad$ Reduced frequency number, $k=\omega R^{*} / \bar{a}$

$k_{\text {eff }} \quad$ Effective thermal conductivity of the porous media

$M_{0} \quad$ Steady flow Mach number

$\bar{M} \quad$ Mean Mach number of steady flow, $M_{0}=\frac{s^{2}}{8 \gamma} \frac{d p_{0}}{d \xi}\left(1-\eta^{2}\right)$

p $\quad$ Acoustic pressure

$p_{0} \quad$ Steady flow pressure

$R \quad$ Radius of capillary duct

$R_{0} \quad$ Gas constant

$s \quad$ Shear wave number

$t^{*} \quad$ Time

$T \quad$ Temperature

$u^{*}, v^{*} \quad$ The velocity components in $x$-and $y$-directions

$u, v$

Acoustic velocity components in $x$-and $y$-directions

$x^{*}, r^{*} \quad$ Axial and normal coordinates

$W \quad$ Phase velocities

w dimensionless phase velocity

\section{Greek symbols:}

$\begin{array}{ll}\alpha & \text { Perturbation parameter } \\ \rho & \text { Acoustic density } \\ \bar{\rho} & \text { Mean steady flow density } \\ \rho^{*} & \text { Fluid density } \\ \sigma & \text { Prandtl number } \\ \xi & \text { Dimensionless axial coordinate } \\ \eta & \text { Dimensionless normal coordinate } \\ \gamma & \text { Ratio of specific heats } \\ \omega & \text { Harmonic disturbance frequency } \\ \varepsilon & \text { Porosity } \\ \mu & \text { Dynamic viscosity } \\ \Gamma^{\prime} & \text { Propagation constant } \\ \Gamma^{\prime} & \text { Attenuation } \\ \Gamma^{\prime \prime} & \text { Phase shift angle }\end{array}$




\section{INTRODUCTION}

In porous materials such as fibrous and granular, the absorption process of the acoustic wave takes place through viscosity and thermal losses of the acoustic energy inside the micro tubes forming the material. This kind of material is widely used in room acoustics, in order to control reverberation time, to avoid undesired reflections, and to fill double wall cavities, floors and ceilings, etc. If the acoustic improvements are restricted to interior spaces (buildings halls, theaters, dwellings, factories, vehicle cabins, etc.), usually mineral wools or open pore foams can be used. On the other hand, for outdoor problems for instant acoustic noise barriers against traffic noise, the absorption provided by granular materials such as porous concrete should be employed.

The problem of a propagation of sound waves in fluids contained in a plain medium is a classical one, to which famous names are connected like Helmholtz [1], Kirchhoff [2] and Rayleigh [3]. Numerical solutions of the problem were published by Tsao [4], Gerlasch and Parker [5], Scarton and Rouleau [6] and by Shields et al. [7]. It is shown that the two-main parameters governing the propagation of sound waves in gases contained in rigid cylindrical tubes are the shear wave number and the reduced frequency. It is demonstrated that most of the analytical solution are dependent only on the shear wave number and that they are covered completely by the solution for the first time by Zwikker and Kosten [8]. The full solution of the problem has been obtained by Tijdeman [9] in the form of a complicated, complex transcendental equation, then this equation is solved numerically by using Newton-Raphson procedure and results are presented for a wide range of shear wave number and reduced frequency. A first approximation to the effects of mean flow on sound propagation through cylindrical capillary tubes is achieved by Peat [10]. The problem of sound waves propagation in a stationary fluid or flowing fluid in a porous medium is a new one. Wang et al., [11] studied the effects of compression on the sound absorption of porous materials with an elastic frame. Sato and Kanki [12] formulates for compression waves and oscillating flow in circular pipe.

In this manuscript the sound waves propagation in a stationary or flowing fluid in a porous medium is studied. The governing equations are going to be written then the flow is assumed to has a small acoustic disturbance of frequency and the governing equations are transformed using a set of variables in suitable dimensionless form then a solution of the propagation constant is sought for the governing equations for both attenuation and phase shift angle. In this study, a first approximation is sought for the effect of the porous matrix on sound waves propagation in cylindrical tubes. Analytical expressions for the propagation constant of axial wave motion are obtained from variational solutions by using parabolic forms of the velocity profiles. Comparison with previous works available in the limit of plain medium is achieved and shows an excellent agreement.

\section{MATHEMATICAL FORMULATION}

The thermo-viscous effects in the fluid filling interstices among the fibers or the particles are responsible for the energy loss of the propagation acoustic wave. Generally, thermal losses are much lower than viscous losses in this kind of material. For mean flow through a capillary porous duct it is reasonable to apply the conventional boundary layer approximation that the axial velocity is much greater than he radial velocity, 
$u^{*}>>v^{*}$, and the changes in the radial direction occur much rapidly than those in the axial direction. The basic equations which govern acoustic wave propagation in a rigid tube filled with a porous media are the continuity, momentum and energy equations:

$$
\begin{aligned}
& \varepsilon \frac{\partial \rho^{*}}{\partial t^{*}}+u^{*} \frac{\partial \rho^{*}}{\partial x^{*}}+\rho^{*} \frac{\partial u^{*}}{\partial x^{*}}=0 \\
& \rho^{*}\left[\varepsilon^{-1} \frac{\partial u^{*}}{\partial t^{*}}+\varepsilon^{-2} u^{*} \frac{\partial u^{*}}{\partial x^{*}}\right]=-\frac{\partial p^{*}}{\partial x^{*}}-\frac{\mu}{K} u^{*}+\mu \varepsilon^{-1}\left[\left(\frac{\partial^{2} u^{*}}{\partial r^{* 2}}+\frac{1}{r^{*}} \frac{\partial u^{*}}{\partial r^{*}}\right)+\frac{1}{3} \frac{\partial}{\partial x^{*}} \frac{\partial u^{*}}{\partial x^{*}}\right] \\
& \rho^{*} c_{p}\left[\frac{\partial T^{*}}{\partial t^{*}}+u^{*} \frac{\partial T^{*}}{\partial x^{*}}\right]=k_{e f f}\left[\left(\frac{\partial^{2} T^{*}}{\partial r^{* 2}}+\frac{1}{r^{*}} \frac{\partial T^{*}}{\partial r^{*}}\right)\right]+\frac{\partial p^{*}}{\partial t^{*}}+u^{*} \frac{\partial p^{*}}{\partial x^{*}}+\mu\left(\frac{\partial u^{*}}{\partial r^{*}}\right)^{2}
\end{aligned}
$$

where $u^{*}$ is the velocity component in the axial direction. $\rho^{*}, p^{*}$ and $T^{*}$ are the fluid density, pressure and temperature, $\mu$ is the absolute viscosity and $K$ is the permeability and $\varepsilon$ is the porosity of the porous medium, $c_{p}$ and $k_{\text {eff }}$ are the specific heat and effective thermal conductivity of the porous media. The fluid is assumed to be a perfect gas governed by the equation of state:

$p^{*}=\rho^{*} R_{0} T^{*}$

where $R_{0}$ is the gas constant. Next, it is assumed that the flow through the circular duct is a superposition of a fully developed laminar, incompressible, axial steady flow and a small harmonic acoustic disturbance of frequency $\omega$. The steady flow is taken to have constant density $\bar{\rho}$ and a speed of sound $\bar{a}$ such that the fluid variables can be expanded in the form:

$$
\begin{aligned}
& \rho^{*}=\bar{\rho}\left(1+\alpha \rho(\eta) e^{\Gamma \xi} e^{i \omega t^{*}}\right), \\
& u^{*}=\bar{a}\left(M_{0}(\eta)+\alpha u(\eta) e^{\Gamma \xi} e^{i \omega t^{*}}\right), \\
& p^{*}=\left(\overline{\rho a}^{2} / \gamma\right)\left(p_{0}(\xi)+\alpha p(\eta) e^{\Gamma \xi} e^{i \omega t^{*}}\right)
\end{aligned}
$$

and

$$
T^{*}=\left(\bar{a}^{2} / \gamma R_{0}\right)\left(1+\alpha T(\eta) e^{\Gamma \xi} e^{i \omega t^{*}}\right)
$$

where $\alpha<<1$ and $\gamma$ is the ratio of specific heats. It is seen that the steady flow variables $p_{0}$ and Mach number $M_{0}$ together with acoustic variables $\rho, u, p$ and $T$ are dimensionless. Introduce the following variables in the transformations:

$$
\xi=\omega x^{*} / \bar{a} \quad \eta=r^{*} / R
$$

$R$ is the radius of the capillary duct. The axial acoustic wave motion has been assumed to have complex propagation constant $\Gamma$ which can be expanded as:

$$
\Gamma=\Gamma^{\prime}+i \Gamma^{\prime \prime}
$$

where $\Gamma^{\prime}$ represents the wave attenuation per unit distance and $\Gamma^{\prime \prime}$ represents the phase shift over the same distance. The assume forms of the variables, equations (5-8) 
are substituted into the governing equations (1)-(3) and terms of similar order in $\alpha$ equated. It is found that for zeroth order, the steady flow solution, the equations of continuity and radial momentum are identically satisfied, while the axial momentum equation (1) becomes:

$\frac{s^{2}}{\gamma} \frac{d p_{0}}{d \xi}=\frac{1}{\varphi \eta} \frac{d}{d \eta}\left(\eta \frac{d M_{0}}{d \eta}\right)-D a M_{0}$

Here $s=R \sqrt{\bar{\rho} \omega / \mu}$ is the shear wave number, $D a=R^{2} / K$. This equation is similar to the classical equation of Hagen-Poiseuilli flow, the solution of which, with no-slip boundary conditions, gives a parabolic velocity profile:

$M_{0}=\frac{s^{2}}{\gamma} \frac{d p_{0}}{d \xi}\left(\frac{1-\eta^{2}}{4}\right)=2 \bar{M}\left(1-\eta^{2}\right)$

The $\bar{M}$ is the mean Mach number of the steady flow. The linearized acoustic equations follow from equating terms of first order in $\alpha$ in the governing equations:

$$
\begin{gathered}
k\left[\frac{i \rho}{\varepsilon}+\Gamma u+2 \bar{M} \Gamma\left(1-\eta^{2}\right) \rho\right]=0 \\
\frac{i u}{\varepsilon}+\frac{2 \bar{M} \Gamma}{\varepsilon^{2}}\left(1-\eta^{2}\right) u=(-\Gamma / \gamma) p+\left(1 / s^{2} \varepsilon\right)\left[\frac{d^{2} u}{d \eta^{2}}+(1 / \eta) d u / d \eta\right]-\left(D a / s^{2}\right) u \\
i T+2 \bar{M} \Gamma\left(1-\eta^{2}\right) T=\frac{1}{\sigma^{2} s^{2}}\left[\frac{d^{2} T}{d \eta^{2}}+(1 / \eta) d T / d \eta\right]+\left(\frac{\gamma-1}{\gamma}\right)\left[i+2 \bar{M} \Gamma\left(1-\eta^{2}\right) p\right] \\
-8\left(\frac{\gamma-1}{s^{2}}\right) \bar{M} \frac{d}{d \eta}(u \eta)
\end{gathered}
$$

$p=\rho+T$

where $p$ is constant, Furthermore, $\sigma=\sqrt{\mu c_{p} / k_{\text {eff }}}$, the square root of the Prandtl number, and $k=\omega R / \bar{a}$ is the reduced frequency. Note that the $\varepsilon$ and $D a$ will reflect the effect of the porous matrix size on the acoustic problem under consideration. The case of $\varepsilon=1$ or $D a=0$ corresponds to the plain medium without the presence of the solid matrix and any values of $0<\varepsilon<1$ or $D a>0$ represent a porous medium with different pore spaces. For the case of $\varepsilon=1$ and $D a=0$, the governing equations (13-15) reduces to those obtained by Peat [10] for the case of a pure plain medium. The no-slip boundary condition of the fluid velocity at wall:

$u=0, T=0$ at $\eta=1$

Since the steady flow profile is also parabolic, variational solutions with the following form of axial acoustic velocity variation is sought:

$u=C\left(1-\eta^{2}\right) \quad$ where $C$ is constant 


\section{VARIATIONAL SOLUTIONS}

A variational solution of the momentum equation results in expression for constant $\mathrm{C}$ :

$$
\begin{aligned}
\frac{\partial f}{\partial u} & =\frac{d}{d \eta}\left(\frac{\partial u}{\partial(d u / d \eta)}\right) \\
f & =\left[\left(1 / s^{2} \varepsilon\right) \eta\left(\frac{d u}{d \eta}\right)^{2}+\frac{i u^{2} \eta}{\varepsilon}+\left(\frac{2 \Gamma}{\gamma}\right) p u \eta+\frac{2 \bar{M} \Gamma}{\varepsilon^{2}}\left(1-\eta^{2}\right) \eta u^{2}+\frac{2 D a}{s^{2}} u^{2} \eta\right] d \eta
\end{aligned}
$$

The best approximation to equation (20) corresponds to the minimum of the functional:

$$
F=\int_{0}^{1}\left[\left(1 / s^{2} \varepsilon\right) \eta\left(\frac{d u}{d \eta}\right)^{2}+\frac{i u^{2} \eta}{\varepsilon}+\left(\frac{2 \Gamma}{\gamma}\right) p u \eta+\frac{2 \bar{M} \Gamma}{\varepsilon^{2}}\left(1-\eta^{2}\right) \eta u^{2}+\frac{D a}{s^{2}} u^{2} \eta\right] d \eta
$$

Thus, the assumed form of trial solution for $u$, equation (18), is substituted into this expression and the minimum is found by setting:

$\partial F / \partial C=0$

and so:

$$
C=-\frac{p \Gamma}{2 \gamma} /\left(\frac{2}{s^{2} \varphi}+\frac{i}{3}+\frac{\bar{M} \Gamma}{2 \varphi^{2}}+\frac{D a}{3 s^{2}}\right)
$$

It is necessary here to consider the equations of energy and state to relate the pressure to the density whilst eliminating the temperature, the energy equation corresponds to the minimum of the functional:

$$
H=\int_{0}^{1}\left[\begin{array}{l}
\left(1 / s^{2} \sigma^{2}\right) \eta\left(\frac{d T}{d \eta}\right)^{2}+i u^{2} \eta+2 \bar{M} \Gamma \eta\left(1-\eta^{2}\right) \eta T^{2}- \\
2\left(\frac{\gamma-1}{\gamma}\right)\left[i+2 \bar{M} \Gamma\left(1-\eta^{2}\right)\right] \eta p T+\frac{16}{s^{2}}(\gamma-1) \bar{M} \eta \frac{d}{d \eta}(\eta u)
\end{array}\right] d \eta
$$

If one assumes a trial solution of the temperature of the form:

$T=D\left(1-\eta^{2}\right), D$ is constant

Which identically satisfies the boundary conditions on the temperature, then the minimum of the functional is found by setting:

$$
\partial H / \partial D=0
$$

An expression for the constant results; namely: 


$$
D=\left(\frac{\gamma-1}{\gamma}\right)\left(\frac{i}{2}+\frac{2}{3} \bar{M} \Gamma\right) p /\left(\frac{2}{s^{2} \sigma^{2}}+\frac{i}{3}+\frac{\bar{M} \Gamma}{2}\right)
$$

Now from equations (16) and (27),

$$
\rho=p-D\left(1-\eta^{2}\right)
$$

And hence the integral form of the continuity equation (23) becomes:

$$
\int_{0}^{1} \eta\left[\frac{i}{\varphi}+2 \bar{M} \Gamma\left(1-\eta^{2}\right)\left[p-D\left(1-\eta^{2}\right)\right]+\Gamma C\left(1-\eta^{2}\right)\right] d \eta=0
$$

Evaluating the integral and substituting from equation (23) and equation (27) for $C$ and $D$ leads to an equation for the propagation constant:

$$
\frac{i}{\varepsilon}+\bar{M} \Gamma-\frac{\frac{\Gamma^{2}}{4 \gamma}}{\left(\frac{2}{s^{2} \varepsilon}+\frac{i}{3 \varepsilon}+\frac{D a}{3 s^{2}}+\frac{\bar{M} \Gamma}{2 \varepsilon^{2}}\right)}-\frac{\left(\frac{\gamma-1}{\gamma}\right)\left[\frac{i}{2}+\frac{2 \bar{M} \Gamma}{3}\right]\left[\frac{i}{2 \varepsilon}+\frac{2 \bar{M} \Gamma}{3}\right]}{\left(\frac{2}{\sigma^{2} \varepsilon^{2}}+\frac{i}{3}+\frac{\bar{M} \Gamma}{2}\right)}+=0
$$

This is a cubic equation in $\Gamma$ for which no single analytical solution can be fund, in the two limiting cases of either zero steady flow $\bar{M}=0$, or fluid of unit Prandtl number, $\sigma=1$, the equation becomes quadratic in $\Gamma$ and an analytical solution results.

\section{RESULTS AND DISCUSSION}

In the limit of steady flow $\bar{M}=0.0$, comparison of variational solution with Peat [10] and Zwicker and Kosten [8] in the limits of plain medium $\varepsilon=1$ and $D a=0$ is shown in table.

1. Large value of $s=R \sqrt{\bar{\rho} \omega / \mu}$ can be obtained not only for large tube radii but also for high frequencies, large mean densities or pressure and small viscosity. Attention is given now to the imaginary part of propagation constant $\Gamma^{\prime \prime}$, the greatest physical insight follows from a study of phase velocity $W$, which when written in non-dimensional form is simply the inverse of $\Gamma^{\prime \prime}$, or

$$
w=|W / \bar{a}|=\left|1 / \Gamma^{\prime \prime}\right|
$$

Figure1 is a plot of the modulus of wave attenuation per unit distance, $\Gamma^{\prime}$ and phase velocity $\left|1 / \Gamma^{\prime \prime}\right|$ for varying shear wave number and Mach numbers. It is clear that as the Mach number is increased the attenuation for the forward and hydrodynamic sound waves is decreased and consequently the phase velocities are increased; thi is due to unfavorable collision effect at higher velocities of the forward and hydrodynamic sound waves, while the attenuation is decreased for the backward sound wave and consequently the phase velocities are decreased; this is due to favorable mean flow of retarding the backward sound waves. 
Figure 2 is a plot of the modulus of wave attenuation per unit distance, $\Gamma^{\prime}$ and phase velocities $\mid 1 / \Gamma^{\prime \prime}$ for varying shear wave number and porosity, it is found that as the porosity is increased the attenuation is decreased and the phase velocities are increased for all forward, backward and hydrodynamic sound waves; this is due to neglecting porous medium effects in damping sound waves, as we move toward the plain media limit.

Figure 3 shows the effect of Darcy number on both attenuation and phase velocities. It is clear that as the Darcy number is increased the attenuation is increased for forward, backward and hydrodynamic sound waves; this is due favorable damping effect of the solid matrix of the three types of sound waves. It is also found that as the Darcy number is increased the phase velocities are increased for the forward, backward and hydrodynamic sound waves; this is due to favorable effect of the porous matrix in increasing phase shift of the three sound waves.

\section{CONCLUSIONS}

It is found that the inclusion of the solid matrix increases attenuation and decreases phase velocities for all the forward, backward and hydrodynamic sound waves; this is due to favorable retarding effect of the solid matrix on the propagating sound waves.

\section{REFERENCES}

1. H. V. Helmholz, Verhandlung der Naturhistorish-Medizinischen Vereins zu Heidelberg, Bd III, vol. 16, 1863.

2. G. Kirchhoff, Uber den Einfluss der Waermeleitung in einem Gas auf den Schallbewegung, Proggendorfer Annalen, vol. 134, pp. 177-193, 1868.

3. Lord Rayleigh, Theory of sound, volume II, London: The Macmillan Company second edition, pp. 319-326, 1896.

4. S. Tsao, Numerical solutions of transients in pneumatic networks-transmission line calculations, Journal of Applied Mechanics, Transactions of American Society of Mechanical Engineers, vol. 35, pp. 588-595, 1968.

5. C. R. Gerlach and J. D. Parker, Wave propagation in viscous fluid lines including higher mode effects, Journal of Basic Engineering, Transactions of the American Society of Mechanical engineers, vol. 89, pp. 782-788, 1976.

6. H. A. Scarton and W. T. Rouleau, Axisymetric waves in compressible Newtonian liquids contained in rigid tubes: steady periodic mode shapes and dispersion by method of eignvalues, Journal of Fluid Mechanics, vol. 58, pp. 595-621, 1973.

7. F. d. Shields, K. P. Lee and W. J. Wiley, Numerical solution for sound velocity and absorbing in cylindrical tubes, Journal of the Acoustical Society of America, vol. 37, pp. 724-729, 1965.

8. C. Zwikker and C. Kosten, Sound absorbing materials, Amsterdam: Elsevier, 1949.

9. H. Tijdeman, On the propagation of sound waves in cylindrical tubes, Journal of Sound and Vibration, vol. 162, pp. 1-33, 1975.

10. K. S. Peat, A first approximation to the effects of mean flow on sound flow on sound propagation through cylindrical capillary tubes, Journal of sound and vibration, vol. 175, no. 4, pp. 475-489, 1993. 
11. C.-N., Wang, Y.-M., Kuo and S.-K., Chen, Effects of compression on the sound absorption of porous materials with an elastic frame, Applied Acoustics, vol. 69, pp. 31-39, 2008.

12. Y. Sato, and $\mathrm{H}$. Kanki, Formulas for compression wave and oscillating flow in circular pipe, Applied Acoustics, vol. 69, pp. 1-11, 2008.

Table 1

Attenuation , $\left|\Gamma^{\prime}\right|$, for the case $M=0$

\begin{tabular}{|c|c|c|c||c|c|c|c|}
\hline $\begin{array}{c}\text { Shear wave } \\
\text { number, s }\end{array}$ & $\begin{array}{c}\text { Peat } \\
{[\mathbf{1 0 ]}}\end{array}$ & $\begin{array}{c}\text { Zwikker } \\
\text { and } \\
\text { Kosten [8] }\end{array}$ & Present & $\begin{array}{c}\text { Shear } \\
\text { wave } \\
\text { number, s }\end{array}$ & $\begin{array}{c}\text { Peat } \\
{[\mathbf{1 0 ]}}\end{array}$ & $\begin{array}{c}\text { Zwikker } \\
\text { and } \\
\text { Kosten [8] }\end{array}$ & Present \\
\hline 0.2 & 11.797 & 11.797 & 11.797 & 3.0 & 0.494 & 0.521 & 0.494 \\
\hline 0.4 & 5.846 & 5.846 & 5.846 & 3.2 & 0.447 & 0.477 & 0.447 \\
\hline 0.6 & 3.840 & 3.840 & 3.840 & 3.4 & 0.406 & 0.440 & 0.406 \\
\hline 0.8 & 2.821 & 2.822 & 2.822 & 3.6 & 0.370 & 0.408 & 0.370 \\
\hline 1.0 & 2.199 & 2.200 & 2.200 & 3.8 & 0.339 & 0.380 & 0.339 \\
\hline 1.2 & 1.776 & 1.779 & 1.777 & 4.0 & 0.312 & 0.356 & 0.311 \\
\hline 1.4 & 1.469 & 1.473 & 1.470 & 4.2 & 0.287 & 0.334 & 0.286 \\
\hline 1.6 & 1.236 & 1.242 & 1.237 & 4.4 & 0.265 & 0.315 & 0.264 \\
\hline 1.8 & 1.054 & 1.062 & 1.055 & 4.6 & 0.246 & 0.298 & 0.245 \\
\hline 2.0 & 0.909 & 0.919 & 0.909 & 4.8 & 0.228 & 0.283 & 0.227 \\
\hline 2.2 & 0.791 & 0.804 & 0.792 & 5.0 & 0.212 & 0.269 & 0.211 \\
\hline 2.4 & 0.695 & 0.712 & 0.696 & 6.0 & 0.152 & 0.216 & 0.151 \\
\hline 2.6 & 0.616 & 0.636 & 0.616 & 7.0 & 0.113 & 0.181 & 0.112 \\
\hline 2.8 & 0.550 & 0.573 & 0.550 & 8.0 & 0.087 & 0.156 & 0.087 \\
\hline
\end{tabular}



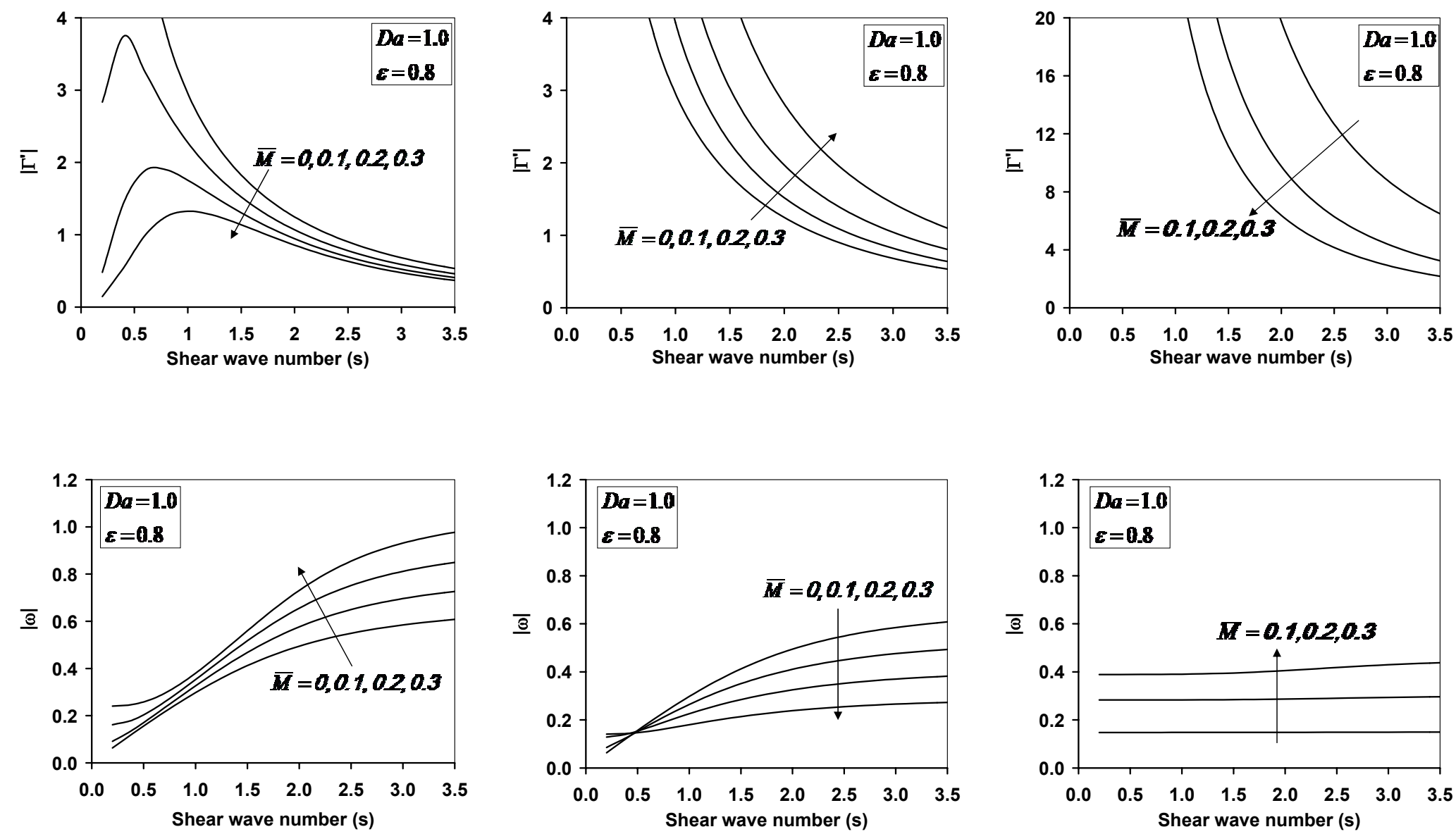

Fig. 1 The attenuation and phase velocities for forward, backward and hydrodynamic sound waves for different values of Mach numbers. 

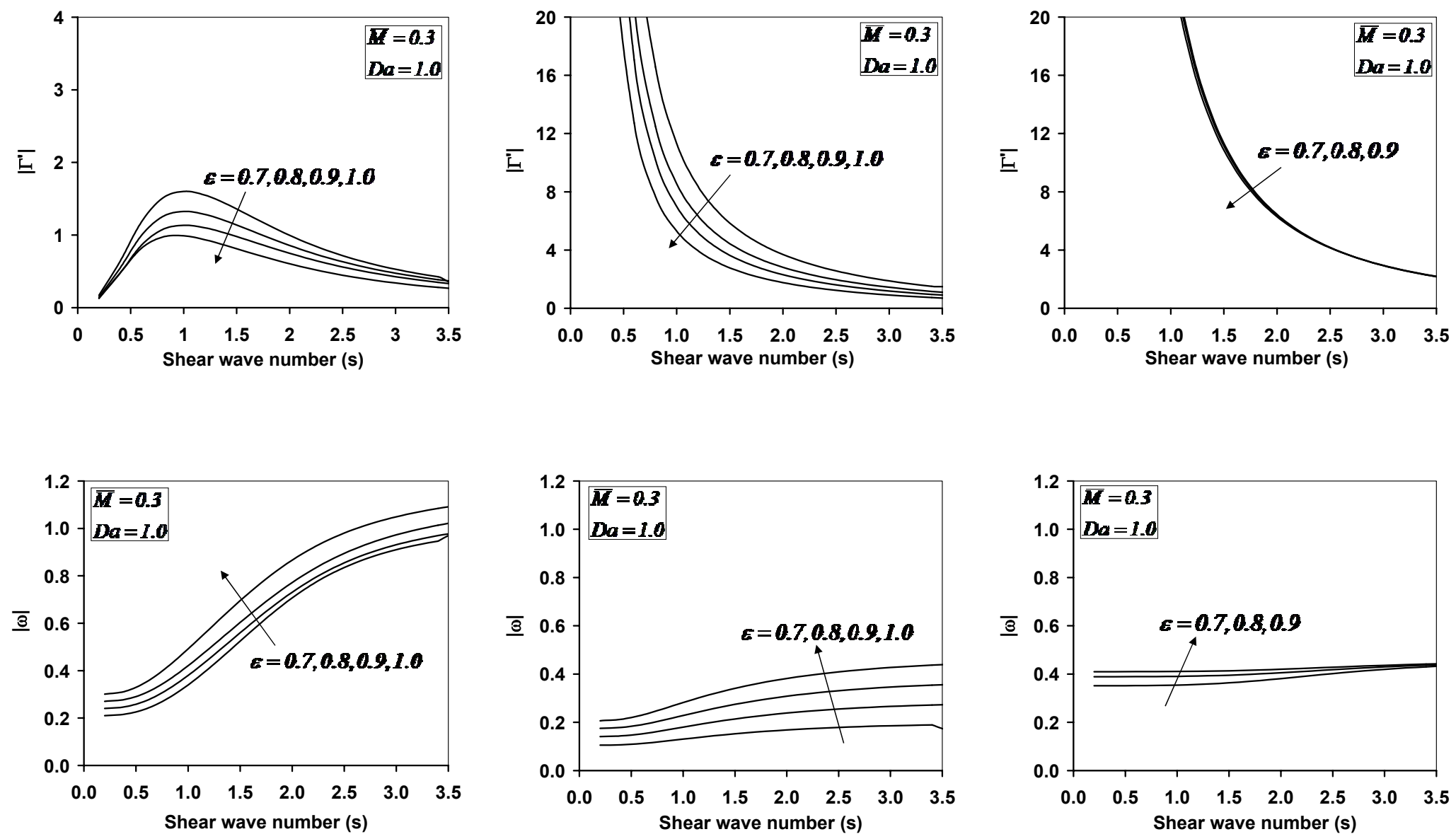

Fig. 2 The attenuation and phase velocities for forward, backward and hydrodynamic sound waves for different values of porosity and For $\mathrm{M}=0.3$ and $D a=$ 0.1 , except for $D a=0, \varepsilon=1.0$ 

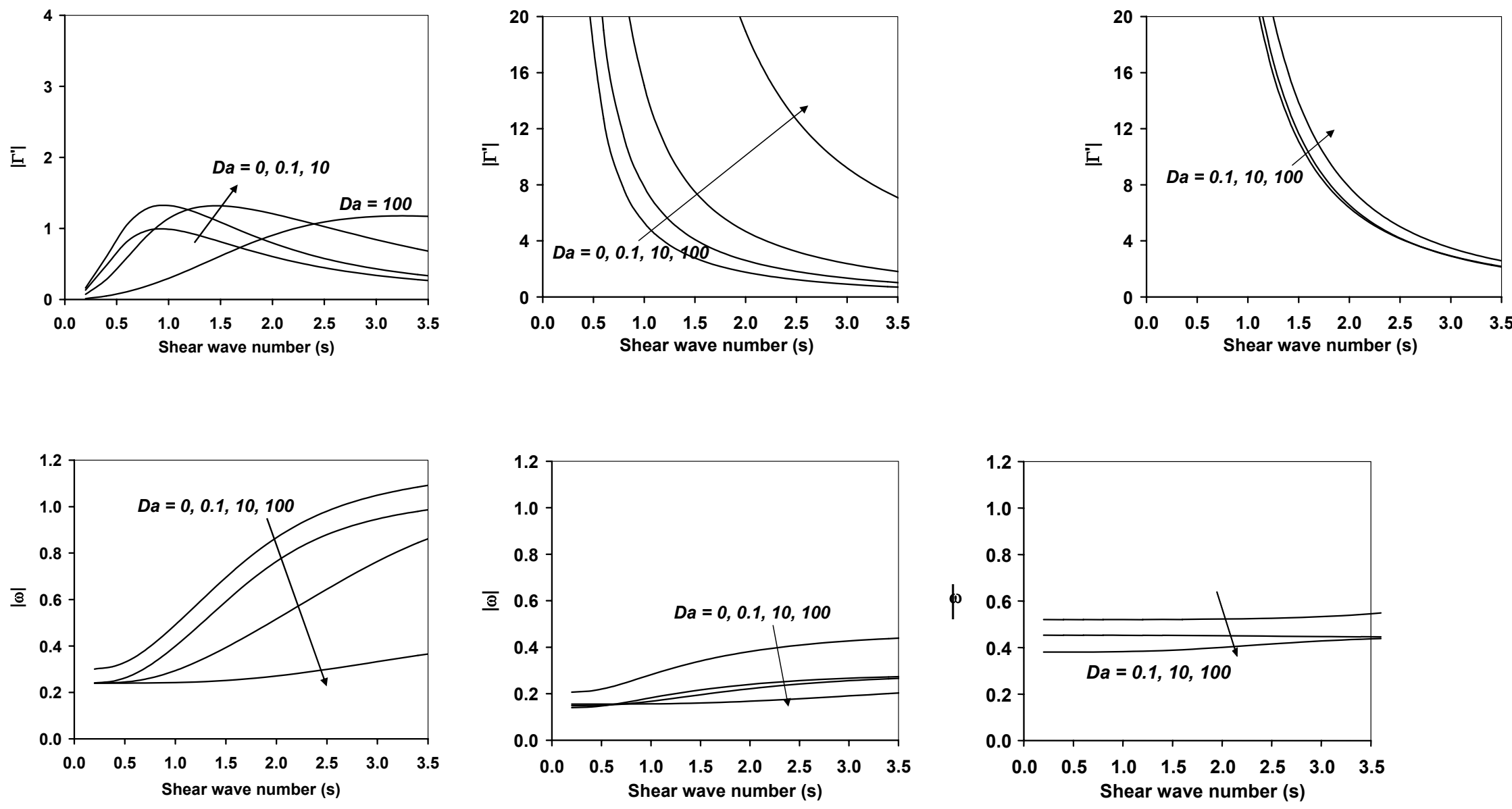

Fig. 3 The attenuation and phase velocities for forward, backward and hydrodynamic sound waves for different values of Darcy numbers and for $M=0.3$ and $\varepsilon$ $=0.8$, except for $D a=0, \varepsilon=1.0$ 